\title{
An Introduction to Golay Complementary Sequences
}

\author{
Elana Kalashnikov \\ Department of Mathematics, University of Alberta
}

\section{INTRODUCTION}

Golay Complementary Sequences were first introduced by Marcel Golay in 1949, but were formally defined in his 1960 article "Complementary Series" [2]. They have many applications: Golay used them in infrared multislit spectrometry, and more recently, they have been applied to Orthogonal Frequency-Division Multiplexing [19]. Aside from the applications, they are of mathematic interest because of the "deep seated symmetries which characterize them," [2] and because of the challenge they present in both constructing them and enumerating them. There are still many open questions about both Golay sequences and their extensions. In this paper, I will discuss the development of Golay sequences over the last decades, and present the main results.

First, I will discuss Golay sequences as Golay himself defined them, presenting his results on their lengths and his direct and recursive constructions. I will then discuss the broadest generalization yet defined, Golay array pairs. It is fruitful to understand complementary sequences as a special case of Golay array pairs, rather than Golay array pairs simply as an extension of the more fundamental complementary sequences. First, I will discuss their basic properties, and then the ways in which they can be transformed into higher or lower dimensional arrays. Secondly, within the context of Golay array pairs, I will discuss the other main generalizations and developments, beginning with Jedwab and Davis' non-recursive structure, which led to the division of standard and non-standard Golay sequences, both of which I will discuss. Thirdly, I will present the basic extensions of Golay sequences: Golay sets, and multiple L-shift complementary sequences.

\section{ORIGINAL GOLAY SEQUENCES}

\subsection{Definitions}

Golay defined his "complementary series", which we call sequences, in his 1961 paper ([2]) in three equivalent ways. A proof of equivalence will follow the definitions.

For the first definition, we need the notion of a separation of a sequence. A separation of length $j$ of sequence $a=\left(\begin{array}{llll}a_{0} & a_{1} & \cdots & a_{n-1}\end{array}\right)$, are the pairs $\left(a_{i}, a_{i+j}\right)-$ that is, pairs of elements of the sequence separated by $j$.

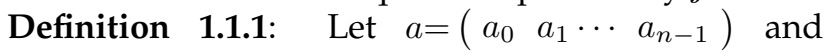
$b=\left(\begin{array}{llll}b_{0} & b_{1} & \cdots & b_{n-1}\end{array}\right)$ be two length $n$ sequences with $a_{k}, \quad b_{k} \in\{-1,1\} \forall 0 \leq k \leq n-1$. Then $(a, b)$ are a Go- lay complementary pairs if, under any given separation $j$, the number of like pairs in $a$ is equal to the number of unlike pairs in $b . a$ and $b$ are then Golay sequences.

Example 1.1.2: $\left(\begin{array}{llll}1 & 1 & -1 & 1\end{array}\right)$ and $\left(\begin{array}{llll}1 & 1 & 1 & -1\end{array}\right)$ are a Golay complementary pair.

This definition, however, cannot easily accommodate important extensions (such as Golay sequences in nonbinary alphabets). Thus, the second definition,

Definition 1.1.3: Let $a=\left(\begin{array}{lll}a_{0} & a_{1} \cdots a_{n-1}\end{array}\right)$ and $b=\left(\begin{array}{llll}b_{0} & b_{1} & \cdots & b_{n-1}\end{array}\right)$ be two length $n$ sequences with $a_{k}, \quad b_{k} \in\{-1,+1\} \forall 0 \leq k \leq n-1$. Let

$$
C_{a}(j):=\sum_{k=0}^{n-1-j} a_{k} a_{k+j}
$$

Then $(a, b)$ are a Golay complementary pair if for every $0<j \leq n-1$.

$$
C_{a}(j)+C_{b}(j)=0
$$

For $a=\left(\begin{array}{llll}a_{0} & a_{1} & \cdots & a_{n-1}\end{array}\right)$ a length $n$ sequences, the polynomial in $z \in \mathbb{C}$ associated with $a$ is $a(z)=$ $\sum_{k=0}^{n-1} a_{k} z^{k}$ This leads to a third definition of the original Golay Complementary Pairs.

Definition 1.1.4: Let $a=\left(\begin{array}{lll}a_{0} & a_{1} \cdots a_{n-1}\end{array}\right)$ and $b=\left(\begin{array}{llll}b_{0} & b_{1} & \cdots & b_{n-1}\end{array}\right)$ be two length $n$ sequences with $a_{k}, \quad b_{k} \in\{-1,+1\} \forall 0 \leq k \leq n-1$. Then $(a, b)$ are a Golay complementary pair if

$$
a(z) a\left(z^{-1}\right)+b(z) b\left(z^{-1}\right)=2 n
$$

For a sequence $a$, let $o_{a j}$ be the number of unlike pairs, and $e_{a j}$ the number of like pairs, of elements of sequence $a$ under separation $j$ for $0<j \leq n-1$.

These three definitions are equivalent, and are useful in different contexts. The first definition is primarily useful as an intuitive way of seeing the properties of such pairs of sequences, without formulas.

Proposition 1.1.5: Definitions 1.1.1, 1.1.3, and 1.1.4 are equivalent

Proof: Let $a=\left(\begin{array}{lll}a_{0} & a_{1} \cdots a_{n-1}\end{array}\right)$ and $b=$ $\left(\begin{array}{llll}b_{0} & b_{1} & \cdots & b_{n-1}\end{array}\right)$ be two length $n$ sequences with $a_{k}, \quad b_{k} \in\{-1,+1\} \forall 0 \leq k \leq n-1$. Assume Definition 1. By assumption, there are $o_{a j}$ like pairs for sequence $b$ under the same separation, and $n-1-j-o_{a j}$ unlike pairs of sequence $b$. There are $n-1-j-o_{a j}$ like pairs for sequence $a$. Then

$$
\begin{gathered}
\sum_{k=0}^{n-1-j} a_{k} a_{k+j}+\sum_{k=0}^{n-1-j} b_{k} b_{k+j} \\
=\left(n-1-j-o_{a j}-o_{a j}\right)+\left(o_{a j}-\left(n-1-j-o_{a j}\right)\right)=0
\end{gathered}
$$


So $(a, b)$ are a Golay pair by Definition 3. Now note that $a(z) a\left(z^{-1}\right)$

$$
\begin{array}{lr}
= & \left(\sum_{k=0}^{n-1} a_{k} z^{k}\right)\left(\sum_{k=0}^{n-1} a_{k} z^{-k}\right) \\
= & \sum_{k=0}^{n-1} a_{k}^{2}+\sum_{j=1}^{n-1} \sum_{k=0}^{n-1-j} a_{k} a_{k+j}\left(z^{j}+z^{-j}\right) \\
= & n+\sum_{j=1}^{n-1} \sum_{k=0}^{n-1-j} a_{k} a_{k+j}\left(z^{j}+z^{-j}\right)
\end{array}
$$

Thus,

$$
\begin{array}{cc} 
& a(z) a\left(z^{-1}\right)+b(z) b\left(z^{-1}\right) \\
= & n+\sum_{j=1}^{n-1} \sum_{k=0}^{n-1-j} a_{k} a_{k+j}\left(z^{j}+z^{-j}\right) \\
+ & n+\sum_{j=1}^{n-1} \sum_{k=0}^{n-1-j} b_{k} b_{k+j}\left(z^{j}+z^{-j}\right) \\
= & 2 n+\sum_{j=1}^{n-1} \sum_{k=0}^{n-1-j}\left(a_{k} a_{k+j}+b_{k} b_{k+j}\right)\left(z^{j}+z^{-j}\right) \\
= &
\end{array}
$$

So $a$ and $b$ satisfy Definition 4 .

Now suppose that $a$ and $b$ are a Golay pair by Definition 4. I will show that this means they are a Golay Pair by Definition 1. As we see from above, if $a(z) a\left(z^{-1}\right)+b(z) b\left(z^{-1}\right)=2 n$, then

$$
2 n+\sum_{k=1}^{n-1} \sum_{j=0}^{n-1-j}\left(a_{k} a_{k+j}+b_{k} b_{k+j}\right)\left(z^{j}+z^{-j}\right)=2 n
$$

So

$\sum_{j=0}^{n-1-j}\left(a_{k} a_{k+j}+b_{k} b_{k+j}\right)\left(z^{j}+z^{-j}\right)=0$ for all $z \in C$

which implies that

$$
\begin{array}{rr}
\sum_{k=0}^{n-1-j} a_{k} a_{k+j}+ & \sum_{k=0}^{n-1-j} b_{k} b_{k+j} \\
= & n-1-o_{a j}-o_{a j}+e_{b j}-\left(n-1-e_{b j}\right) \\
= & 0 \text { for every } 0<j \leq n-1
\end{array}
$$

Thus, $o_{a j}=e_{b j}$, so $a$ and $b$ are a Golay pair by Definition 1.

\subsection{Lengths for which Golay Sequences exist}

Golay sequences can only exist for certain lengths, a fact which may be somewhat surprising at first glance.
While a chief difficulty is finding Golay sequences, it is helpful to find some necessary conditions on the lengths of Golay sequences. Thus, as part of the project of discovering which length Golay sequences exist, we will present some basic restrictions on possible lengths.

Proposition 1.2.1: If $(a, b)$ are a Golay pair of length $n$, then

a. $n$ is the sum of two integer squares [2]

b. $n$ is even [2]

c. $n$ is not divisible by integer $p$, where $p$ is a prime number and $p \equiv 3 \bmod 4$ [17].

Proof: Let $a$ and $b$ be a Golay complementary pair.

a. Using definition 1.1.4 of a Golay complementary pair, we find

$$
\begin{gathered}
a(z) a\left(z^{-1}\right)+b(z) b\left(z^{-1}\right)=2 n \\
a(1)^{2}+b(1)^{2}=2 n \\
\left(\frac{a(1)+b(1)}{2}\right)^{2}+\left(\frac{a(1)-b(1)}{2}\right)^{2}=n
\end{gathered}
$$

$a(1)=o_{a 1}(1+(-1))+n_{1}(1+1)+$ $n_{2}(-1+(-1))=2\left(n_{1}-n_{2}\right)$, where $n_{1}+n_{2}=$ $n-1$, and $n_{1}, n_{2} \in \mathbb{Z}$ and positive. Similarly for $b(1)$. Thus

$$
\frac{a(1)+b(1)}{2}, \frac{a(1)-b(1)}{2} \in \mathbb{Z}
$$

So this gives us that the length of a Golay sequence must be the sum of two integer squares.

b. From [16], [5],

$$
o_{a j}+e_{a j}=n-j
$$

and

$$
C_{a}(j)=e_{a j}-o_{a j}
$$

and

$$
\prod_{k=0}^{n-1-j} a_{k} a_{k+j}=(-1)^{o_{a}}=(-1)^{\left(n-j-C_{a}(j)\right) / 2}
$$

$$
\begin{aligned}
& \prod_{k=0}^{n-2} a_{k} a_{k+1} \prod_{k=0}^{0} a_{k} a_{k+n-1} \\
& =\left(a_{0} a_{1}\right)\left(a_{1} a_{2}\right)\left(a_{2} a_{3}\right) \cdots\left(a_{n-2} a_{n-1}\right)\left(a_{0} a_{n-1}\right) \\
& =a_{0}^{2} a_{1}^{2} \cdots a_{n-1}^{2}=1
\end{aligned}
$$




$$
\begin{aligned}
& =(-1)^{\left(n-1-C_{a}(1)\right) / 2}(-1)^{\left(n-(n-1)-C_{a}(n-1)\right) / 2} \\
& =(-1)^{\left(n-C_{a}(1)-C_{a}(n-1)\right) / 2} \\
& \quad C_{a}(1)+C_{a}(n-1)=n(\bmod 4)
\end{aligned}
$$

This also holds for $b$, the Golay complementary sequence which pairs with $a$. Thus

$C_{a}(1)+C_{a}(n-1)+C_{b}(1)+C_{b}(n-1)=0=2 n(\bmod 4)$

So $n$ is even.

c. Because of its length, the proof of this is not included. Refer to [17].

\subsection{Recursive Methods of Producing Golay Sequences}

Golay sequences, however, are useful only if they exist. Necessary conditions on the lengths of Golay sequences do not help with producing them. One way to actually find Golay sequences is to produce them from already known Golay sequences, that is, recursively. Below, I begin by reviewing some operations on sequences in order to make notation simpler when I discuss the recursive methods.

Let $a=\left(\begin{array}{llll}a_{0} & a_{1} & \cdots & a_{n-1}\end{array}\right)$ be a length $n$ sequence, $b=$ $\left(\begin{array}{llll}b_{0} & b_{1} & \cdots & b_{m-1}\end{array}\right)$ a length $m$ sequence.

- Multiplication by a constant: $c a:=\left(\begin{array}{cccc}c a_{0} & c a_{1} \cdots & c a_{n-1}\end{array}\right)$, for $c$ a constant

- Reversal: $\tilde{a}:=\left(\begin{array}{llll}a_{n-1} & a_{n-2} & \cdots & a_{0}\end{array}\right)$

- Altering: $\check{a}:=\left((-1)^{0} a_{0}(-1)^{1} a_{1} \cdots(-1)^{n-1} a_{n-1}\right)$

- Concatenation:

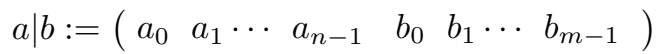

- Interleaving: $a \sim b:=\left(\begin{array}{lllllll}a_{0} & b_{0} & a_{1} & b_{1} & \cdots & a_{n-1} & b_{m-1}\end{array}\right)$

- Tensor product: $a \otimes b:=\left(\begin{array}{llll}a_{0} b & a_{1} b & \cdots & a_{n-1} b\end{array}\right)$ a sequence of length $m n$

- Addition (take $m=n$ ): $a+b:=\left(\begin{array}{lll}a_{0}+b_{0} & a_{1}+b_{1} \cdots a_{n-1}+b_{n-1}\end{array}\right)$

Result 1.3.1 Let $a=\left(\begin{array}{llll}a_{0} & a_{1} \cdots a_{n-1}\end{array}\right)$ and $b=$ $\left(\begin{array}{llll}b_{0} & b_{1} & \cdots & b_{n-1}\end{array}\right)$ be two length $n$ sequences that form a Golay Complementary pair. Then reversing, negating (multiplying by $c=-1$ ), or altering either $a$ or $b$, or both, still gives a Golay pair. Also, pairs $(a|b, a|(-b))$ and $(a \sim b, a \sim(-b))$ form Golay pairs of length $2 n$.
Thus, from Golay sequences of length $n$, we can find Golay sequences of length $2 n$. These basic methods allow us quickly extend the number of known Golay sequences.

Theorem 1.3.2 Let $(a, b)$ and $(c, d)$ be Golay pairs of length $n$ and length $m$ respectively. Then $(e, f)$ form a Golay pair of length $m n$, where

$e(z)=\frac{a\left(z^{m}\right)(c(z)+d(z))+z^{m(n-1)} b\left(z^{-m}\right)(c(z)-d(z))}{2}$

$f(z)=\frac{b\left(z^{m}\right)(c(z)+d(z))+z^{m(n-1)} a\left(z^{-m}\right)(c(z)-d(z))}{2}$

Alternatively, using tensor products,

$$
e=\frac{1}{2}[(c+d) \otimes a+(c-d) \otimes \tilde{b}]
$$

and

$$
f=\frac{1}{2}[(c+d) \otimes b-(c-d) \otimes \tilde{a}]
$$

Proof: Details are omitted, but one should show that $e(z) e\left(z^{-1}\right)+f(z) f\left(z^{-1}\right)=2 n$ by using the above construction.

There are Golay sequence pairs of length 2, 10, and 26 (see [2] for examples). From the above theorem, we then know that there are Golay sequence pairs of length $2^{a} 10^{b} 26^{c}$, for all $a, b, c \in \mathbb{Z}, a, b, c \geq 0$. Giving them explicitly is another problem, however. Golay, in his original paper [2], provided a direct construction using Boolean functions for length $n=2^{m}$, which may be of interest to the reader.

Thus far, we have defined Golay complementary sequences, proven some necessary conditions on the length of such sequences, and given recursive methods for finding more such sequences. Much of this was done by Golay early on. Now we move on to a more general construction.

\section{GOLAY ARRAY PAIRS}

In [10], Fiedler, Jedwab, and Parker argue that a "Golay complementary sequence is naturally viewed as a projection of a multi-dimensional Golay array." A Golay complementary sequence is the simplest example of a Golay array pair (for the explicit relation between arrays and sequences, see Definition 2.1.3). Because this is among the most recent and most significant generalizations, I will use the Golay array structure in order to introduce the other extensions of Golay complementary sequences. A Golay array pair generalizes a Golay sequences both in terms of its dimensions and in terms of the entries of the sequence. 


\subsection{Definitions}

Definition 2.1.1 An $n_{1} \times n_{2} \times \cdots \times n_{r}$ array is an $r$-dimensional matrix with complex entries $\mathcal{A}=$ $\left(A\left[i_{1}, \cdots, i_{r}\right]\right) ; i_{1}, \cdots, i_{r} \in \mathbb{Z}$ where $A\left[i_{1}, \cdots, i_{r}\right]=$ 0 if $\exists j$ such that $i_{j}<0$ or $i_{j} \geq n_{j}$. The in-range entries are $\left\{A\left[i_{1}, \cdots, i_{r}\right] \mid \forall l, 0 \leq i_{l}<n_{l}\right\}$. Often, the inrange entries are constrained to lie in a certain alphabet. If this alphabet is $S_{H}=\left\{1, \mathcal{E}, \ldots, \mathcal{E}^{H-1}\right\}$ for $H$ an even integer and $\mathcal{E}=e^{\frac{2 p \sqrt{-1}}{H}}$, then we say that it is an $\mathrm{H}$-phase array. All in-range entries are of the form $A\left[i_{1}, \cdots, i_{r}\right]=\mathcal{E}^{a\left[i_{1}, \cdots, i_{r}\right]}, a\left[i_{1}, \cdots, i_{r}\right] \in \mathbb{Z}_{H}$. Then $\mathcal{A}^{\prime}=a\left[i_{1}, \cdots, i_{r}\right]$ is the array over $\mathbb{Z}_{H}$ corresponding to $\mathcal{A}$.

For $z$ a complex number, let $\bar{z}$ be its complex conjugate.

Definition 2.1.2 The aperiodic autocorrelation function of an $n_{1} \times n_{2} \times \cdots \times n_{r} H$-phase array is

$$
\begin{aligned}
& \mathcal{C}_{\mathcal{A}}\left(u_{1}, \cdots, u_{r}\right) \\
& =\sum_{i_{1}} \cdots \sum_{i_{r}} A\left[i_{1}, \cdots, i_{r}\right] \overline{A\left[i_{1}+u_{1}, \cdots, i_{r}+u_{r}\right]}
\end{aligned}
$$

Definition 2.1.3 An $n_{1} \times n_{2} \times \cdots \times n_{r}$ Golay array pair (GAP) is two $n_{1} \times n_{2} \times \cdots \times n_{r}$ arrays $\mathcal{A}, \mathcal{B}$ with the property that $\mathcal{C}_{\mathcal{A}}\left(u_{1}, \cdots, u_{r}\right)+\mathcal{C}_{\mathcal{B}}\left(u_{1}, \cdots, u_{r}\right)=$ $0, \forall\left(u_{1}, \cdots, u_{r}\right) \neq(0, \ldots, 0)$. Clearly, if $H=2$ and $r=1$, then an $n_{1}$ Golay array pair is an original Golay complementary sequence pair.

Just as we defined the single variable polynomial associated with a Golay sequence, we define the polynomial associated with the $n_{1} \times n_{2} \times \cdots \times n_{r}$ array $\mathcal{A}=\left(A\left[i_{1}, \cdots, i_{r}\right]\right)$ to be

$$
\mathcal{A}\left(z_{1}, \ldots z_{r}\right)=\sum_{i_{1}} \cdots \sum_{i_{r}}\left(A\left[i_{1}, \cdots, i_{r}\right] z_{1}{ }^{i_{1}} \ldots z_{r}{ }^{i_{r}}\right)
$$

For an analogous reason as that for the original Golay sequences, an equivalent condition for $\mathcal{A}, \mathcal{B}$ (as above) to be a Golay array pair is that $\mathcal{A}\left(z_{1}, \ldots z_{r}\right) \overline{\mathcal{A}\left(z_{1}^{-1}, \ldots z_{r}^{-1}\right)}+$ $\mathcal{B}\left(z_{1}, \ldots z_{r}\right) \overline{\mathcal{B}\left(z_{1}^{-1}, \ldots z_{r}^{-1}\right)}$ be a constant.

Let $\mathcal{A}=\left(A\left[i_{1}, \cdots, i_{r}\right]\right)$ be an $n_{1} \times n_{2} \times \cdots \times n_{r}$ array. If $\mathcal{A}$ is an $H$-phase array, define $\mathcal{A}^{*}=\left(A^{*}\left[i_{1}, \cdots, i_{r}\right]\right)$ where

$$
A^{*}\left[i_{1}, \cdots, i_{r}\right]=\overline{A\left[n_{1}-1-i_{1}, \cdots, n_{r}-1-i_{r}\right]}
$$

If $\mathcal{A}$ is an array over $\mathbb{Z}_{H}$, define

$$
A^{*}\left[i_{1}, \cdots, i_{r}\right]=-A\left[n_{1}-1-i_{1}, \cdots, n_{r}-1-i_{r}\right]
$$

\subsection{Restrictions on Length}

Golay complementary sequences were previously shown to be quite restricted in terms of possible length.
Similarly, there are restrictions on the dimensions of Golay Array Pairs. What about a simpler case: restriction on lengths in the case of $r=1$, that is, polyphase Golay complementary sequences? They are not the same as those for binary Golay sequences. Already with $H=4$ (so that $S=\{1,-1, i,-i\})$, we see that there can be odd length Golay sequences:

Example 2.2.1 [1] Sequences ( $\left.\begin{array}{lllll}1 & 1 & 1 & -i & i\end{array}\right)$ and $\left(\begin{array}{lllll}1 & i & -1 & 1 & -i\end{array}\right)$ form a 4 -phase Golay sequence pair.

In fact, for lengths less than 13, the only (non trivial) lengths for which 4-phase Golay sequences do not exist are lengths 7 and 9 (see [1]).

There is little known about the restrictions on lengths for polyphase sequences (and arrays of any dimension), because of the difficulty of performing effective computer searches at higher lengths.

\subsection{Recursive Means of Producing Golay Array Pairs}

As with Golay complementary sequences, we are concerned with finding Golay array pairs, and one way to do this is to use known pairs to create more. In fact, we can use complementary sequences to create Golay array pairs, and vice versa. The results of this section are taken from [5] and [10], where proofs are also to be found.

Let $n:=n_{1} \times n_{2} \times \cdots \times n_{r} ; m:=m_{1} \times m_{2} \times \cdots \times m_{r} ; i:=$ $i_{1}, i_{2}, \cdots, i_{r} ; j:=j_{1}, j_{2}, \cdots, j_{s}$

Theorem 2.3.1 Suppose there exists binary Golay complementary sequence pairs of lengths $n_{1}, n_{2}, \ldots n_{r}$. Then there exists an $n_{1} \times n_{2} \times \cdots \times n_{r}$ binary Golay array pair.

Because we know that binary Golay complementary sequence pairs exists for all lengths in the form of $2^{a} 10^{b} 26^{c}, a, b, c \in \mathbb{Z}, a, b, c \geq 0$, this theorem gives us that there exists a binary $n_{1} \times n_{2} \times \cdots \times n_{r}$ Golay array pair where $n_{i}=2^{a_{i}} 10^{b_{i}} 26^{c_{i}}, a_{i}, b_{i}, c_{i} \in \mathbb{Z}, a_{i}, b_{i}, c_{i} \geq$ $0, \forall i$.

We also have the converse:

Theorem 2.3.2 Suppose there exists an $n_{1} \times n_{2} \times \cdots \times n_{r}$ binary Golay array pair. Then there exists binary Golay complementary sequence pairs of lengths $n_{1}, n_{2}, \ldots n_{r}$.

This theorem leads to some obvious restrictions on the dimensions of binary Golay array pairs. The product of all the dimensions must either be even or equal to one, and no dimension can have a prime factor congruent to 3 modulo 4.

The next theorems are used in Jedwab's three stage process of producing families of Golay complementary sequences [10].

For a positive integer $n$, let $\mathbf{n}^{(r)}:=\overbrace{n \times \cdots \times n}^{r \text { times }}$.

Theorem 2.3.3 Let $n \geq 1$ be an integer. Then let $x:=x_{1}, x_{2}, \ldots x_{n}$, and for $0 \leq k \leq n$ an integer, let $i_{k}:=i_{k, 1}, i_{k, 2}, \ldots i_{k, r_{k}}$ and $s_{k}:=s_{k, 1} \times s_{k, 2} \times \cdots \times s_{k, r_{k}}$. Suppose that $\mathcal{A}_{k}=\left(a_{k}\left[i_{k}\right]\right), \mathcal{B}_{k}=\left(b_{k}\left[i_{k}\right]\right)$ form a Golay array pair over $\mathbb{Z}_{H}$ of size $s_{k}$. Then the arrays $\mathcal{F}_{n}=\left(f_{n}\left[i_{0}, i_{1}, \ldots, i_{n}, x\right]\right), \mathcal{G}_{n}=\left(g_{n}\left[i_{0}, i_{1}, \ldots, i_{n}, x\right]\right)$ 
form a Golay array pair of size $s_{0} \times s_{1} \times \cdots \times s_{n} \times \mathbf{2}^{(m)}$ over $\mathbb{Z}_{H}$ where

$$
\begin{aligned}
& f_{n}\left[i_{0}, i_{1}, \ldots, i_{n}, x\right] \\
& :=\sum_{k=1}^{n-1}\left(a_{k}\left[i_{k}\right]+a_{k}^{*}\left[i_{k}\right]-b_{k}\left[i_{k}\right]-b_{k}^{*}\left[i_{k}\right]+\frac{H}{2}\right) x_{k} x_{k+1} \\
& +\sum_{k=1}^{n}\left(b_{k-1}^{*}\left[i_{k-1}\right]+b_{k}\left[i_{k}\right]-a_{k-1}\left[i_{k-1}\right]-a_{k}\left[i_{k}\right]\right) x_{k} \\
& +\sum_{k=0}^{n} a_{k}\left[i_{k}\right] \\
& \quad g_{n}\left[i_{0}, i_{1}, \ldots, i_{n}, x\right]:=f_{n}^{\prime}\left[i_{0}, i_{1}, \ldots, i_{n}, x\right]+\frac{H}{2} x_{1}
\end{aligned}
$$

Where $f_{n}^{\prime}\left[i_{0}, i_{1}, \ldots, i_{n}, x\right]$ is $f_{n}\left[i_{0}, i_{1}, \ldots, i_{n}, x\right]$ with $a_{0}\left[i_{0}\right]$ and $a_{0}^{*}\left[i_{0}\right]$ interchanged, and $b_{0}\left[i_{0}\right]$ and $b_{0}^{*}\left[i_{0}\right]$ interchanged.

This theorem gives a way of taking lower dimensional arrays and using them to construct suitable higher dimensional array pairs. It is the first step by which Jedwab produces Golay complementary sequences from the original Golay array pair. From these higher dimensional arrays, the next step is to produce more such pairs using affine offsets.

Theorem 2.3.4 Let $\mathcal{A}=\left(A\left[i_{1}, \cdots, i_{r}\right]\right)$ and $\mathcal{B}=$ $\left(B\left[i_{1}, \cdots, i_{r}\right]\right)$ be an $n_{1} \times n_{2} \times \cdots \times n_{r}$ Golay array pair over $\mathbb{Z}_{H}$. Then for all $e_{k}, e_{0}^{\prime} \in \mathbb{Z}_{H}, \quad k=0,1, \ldots, r$, $\mathcal{A}^{\prime}=\left(A^{\prime}\left[i_{1}, \cdots, i_{r}\right]\right)$ and $\mathcal{B}^{\prime}=\left(B^{\prime}\left[i_{1}, \cdots, i_{r}\right]\right)$ form a Golay array pair, where

$$
\begin{aligned}
& A^{\prime}\left[i_{1}, \cdots, i_{r}\right]=A\left[i_{1}, \cdots, i_{r}\right]+\sum_{k=1}^{r} e_{k} i_{k}+e_{0} \\
& B^{\prime}\left[i_{1}, \cdots, i_{r}\right]=B\left[i_{1}, \cdots, i_{r}\right]+\sum_{k=1}^{r} e_{k} i_{k}+e_{0}^{\prime}
\end{aligned}
$$

This gives us affine offsets of a higher dimensional Golay array pair.

Let $\phi_{j, k}$ be the projection mapping from $r$ dimensions to $r-1$ dimensions which takes an $n_{1} \times n_{2} \times$ $\cdots \times n_{r}$ array $\mathcal{A}=\left(A\left[i_{1}, \cdots, i_{r}\right]\right)$ over $\mathbb{Z}_{H}$ to the $n_{1} \times \cdots \times n_{j-1} \times n_{j+1} \times \cdots \times n_{k-1} \times n_{j} n_{k} \times$ $n_{k+1} \times \cdots \times n_{r}$ array $\phi_{j, k}(\mathcal{A})=\mathcal{B}$, where $B\left[i_{1}, \ldots, i_{j-1}, i_{j+1}, \ldots, i_{k-1}, i_{j}+s_{j} i_{k}, i_{k+1}, \ldots, i_{r}\right]=$ $A\left[i_{1}, \cdots, i_{r}\right]$.

Theorem 2.3.5 Let $\mathcal{A}=\left(A\left[i_{1}, \cdots, i_{r}\right]\right)$ and $\mathcal{B}=$ $\left(B\left[i_{1}, \cdots, i_{r}\right]\right)$ be an $n_{1} \times n_{2} \times \cdots \times n_{r}$ Golay array pair over $\mathbb{Z}_{H}$. Then $\phi_{j, k}(\mathcal{A})$ and $\phi_{j, k}(\mathcal{B})$ are an $n_{1} \times \cdots \times$ $n_{j-1} \times n_{j+1} \times \cdots \times n_{k-1} \times n_{j} n_{k} \times n_{k+1} \times \cdots \times n_{r}$ Golay array pair over $\mathbb{Z}_{H}$.

This theorem allows Golay sequences to be produced from these higher dimensional Golay array pairs and their affine offsets. Fiedler, Jedwab, and Parker additionally prove that the resulting lower dimension array is independent of the order of the projections (of the form in Theorem 2.34). This means that a succession of $r-1$ projection mappings applied to an $r$ dimensional array can be uniquely described by a directed path that is formed by labeling $\mathrm{r}$ vertices $1,2, \ldots, r$, and joining vertices $j$ and $k$ if one of the projection mappings is $\phi_{j, k}$.
Thus, the directed path is

$$
\phi(1) \longrightarrow \phi(2) \longrightarrow \cdots \longrightarrow \phi(r)
$$

Where $\phi$ is a permutation of $\{1,2, \ldots, r\}$.

This is the final step that Fiedler, Jedwab, and Parker present. They use it to generate infinite families of Golay complementary sequences. Of the infinite families of Golay sequences that they generate, there are two types - standard, and nonstandard. Standard Golay sequences arose from the discovery of the connection of Golay sequences to the Reed-Muller code, and were first introduced in [13].

\section{STANDARD GOLAY SEQUENCES}

\subsection{Introduction to OFDM and Peak to Mean Envelope Power Ratio Reduction}

In order to understand the motivation behind the creation of large sets of Golay complementary sequences, we must look at orthogonal frequency-division multiplexing (OFDM) and the PMEPR (peak to mean envelope power ratio) of Golay complementary sequences. OFDM is way of transmitting data using multicarriers which are all orthogonal to each other [8]. The chief practical difficulty of OFDM is that the peak envelope power over $n$ carriers can be as much $n$ times the mean envelope power. It is advantageous to find codes with have a low PMEPR. Codes, generally speaking, are the rules by which a piece of information is encoded. Here, they are chiefly represented as sequences. The code rate is "the ratio of the number of information bits to the number of coded bits" [13].

Suppose there are $n$ carriers. As done in [13], let $a_{i} \in \mathbb{Z}_{H}$ denote the value of the $\mathrm{i}^{t h}$ carrier over a certain symbol period, and $f_{i}$ the frequency of the $\mathrm{i}^{\text {th }}$ carrier. Then $a_{i}(t)$ is constant over the symbol period. The sequence $\left(\begin{array}{llll}a_{0} & a_{1} & \cdots & a_{n-1}\end{array}\right)$ is a codeword for each symbol period. Let $f$ be a constant and $\triangle f$ an integer multiple of the code rate. In order that the $f_{i}$ be orthogonal, they are constrained by the relation $f_{i}=f+i \triangle f$. As before, let $\mathcal{E}=e^{\frac{2 p \sqrt{-1}}{H}}$. The OFDM signal that is transmitted is the real part of

$$
s(t)=\sum_{i=0}^{n-1} \mathcal{E}^{a_{i}(t)+H f_{i} t}
$$

The envelope power $P_{a}(t)$ of the sequence $a=\left(a_{0} a_{1} \ldots a_{n-1}\right)$ is

$$
\begin{aligned}
& P_{a}(t) \\
& =n+\sum_{u \neq 0} \sum_{i} \mathcal{E}^{a_{i}(t)-a_{i+u}(t)-H u \triangle f t} \\
& =n+\sum_{u \neq 0} \mathcal{E}^{-H u \triangle f t} \mathcal{C}_{a}(u)
\end{aligned}
$$

Note that $P_{a}(t)=\left|s_{a}(t)\right|^{2}$ The peak envelope power (PEP)of a sequence $a$ is the supremum of $P_{a}(t)$ over a symbol period. The PMEPR is PEP/ $n$. 
Claim 3.1.1 A sequence which is a member of Golay complementary pair has PMEPR less than 2.

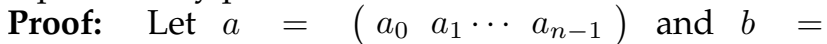
$\left(\begin{array}{llll}b_{0} & b_{1} & \cdots & b_{n-1}\end{array}\right)$ be a Golay complementary pair. So we have $\mathcal{C}_{a}(u)+\mathcal{C}_{b}(u)=0$. Thus,

$P_{a}(t)+P_{b}(t)=2 n+\sum_{u \neq 0} \mathcal{E}^{-H u \triangle f t}\left[\mathcal{C}_{a}(u)+\mathcal{C}_{b}(u)\right]=2 n$

As $P_{a}(t)=\left|s_{a}(t)\right|^{2} \geq 0, P_{b}(t) \leq 2 n$. So the PMEPR of $b$ is less than 2 .

\subsection{The Reed Muller Code and Generalizations}

The Reed Muller Code is an error correcting code. They are high related to Golay complementary sequences, indeed, the Reed Muller Code gives a family of Golay complementary sequences known as Standard Golay Complementary Sequences.

Definition 3.2.1 A Generalized Boolean function is a mapping $\{0,1\}^{m} \rightarrow \mathbb{Z}_{H}$ in $m\{0,1\}$-valued variables $x_{1}, x_{2}, \ldots, x_{m}$. It is easily proved that all generalized Boolean functions can be represented as a $\mathbb{Z}_{H}$ linear combination of the monomials $x_{1}, x_{2}, \ldots, x_{m}, x_{1} x_{2}, \quad x_{1} x_{3}, \ldots, x_{1} x_{2} \cdots x_{m}$. With every Boolean function $f$ there is an associated sequence of length $2^{m}:\left(\begin{array}{llll}f_{0} & f_{1} & \cdots & f_{2^{m}-1}\end{array}\right)$. For a nonnegative integer $i \leq 2^{m}-1$, let $i=\sum_{k=0}^{m-1} i_{k} 2^{k}$, so that $\left(\begin{array}{llll}i_{0} & i_{1} & \cdots & i_{m-1}\end{array}\right)$ is the binary form of $i$. Then $f_{i}=f\left(\begin{array}{llll}i_{0} & i_{1} & \cdots & i_{m-1}\end{array}\right)$ in the vector associated with $f$. Alternatively, given a sequence of length $2^{m}$, its algebraic normal form is the generalized Boolean function associated with it.

Definition 3.2.2 The $r^{\text {th }}$ order generalized Reed Muller code over is the linear code generated (over $\mathbb{Z}_{H}, H$ even) by the Boolean monomials in $x_{1}, x_{2}, \ldots, x_{m}$ of degree at most $r$. It is denoted $R M_{H}(r, m)$.

For a (non-binary) code, the Lee weight of a codeword $a=\left(\begin{array}{llll}a_{0} & a_{1} & \cdots & a_{n-1}\end{array}\right)$ in $\mathbb{Z}_{H}$ is

$$
w t_{L}(a)=\sum_{i=0}^{n-1} \min \left\{a_{i}, H-a_{i} \bmod H\right\}
$$

The Lee distance between two codewords $a$ and $b$ is $d_{L}(a, b)=w t_{L}(a-b \bmod H)$

Theorem 3.2.3 ([4], Theorem 4) The minimum Lee distance of the $R M_{H}(r, m)$ code is $2^{m-r}$.

\subsection{Standard Golay Sequences}

Davis and Jedwab, in [13], were the first to recognize the connection between Golay complementary sequences and the Reed Muller (RM) Codes: that is, standard Golay sequences are cosets of the first order RM Code in the second order RM Code.
Theorem 3.3.1 ([10], Theorem 9) Let $m \geq 1, m \in \mathbb{Z}$, $\phi$ be a permutation of $\{1,2, \ldots, m\}$, and $e_{k}, e_{0}^{\prime} \in \mathbb{Z}_{H}, \quad k=0,1, \ldots, m$. Then the sequences $\mathbf{a}$ and b whose algebraic normal forms are $a\left(x_{1}, x_{2}, \ldots, x_{m}\right)$ and $b\left(x_{1}, x_{2}, \ldots, x_{m}\right)$ respectively form a Golay complementary sequence pair, where

$$
\begin{aligned}
& a\left(x_{1}, x_{2}, \ldots, x_{m}\right) \\
& =\frac{H}{2} \sum_{k=1}^{m-1} x_{\phi(k)} x_{\phi(k+1)}+\sum_{k=1}^{m} e_{k} x_{\phi(k)}+e_{0} \\
& b\left(x_{1}, x_{2}, \ldots, x_{m}\right) \\
& =\frac{H}{2} \sum_{k=1}^{m-1} x_{\phi(k)} x_{\phi(k+1)}+\sum_{k=1}^{m} e_{k} x_{\phi(k)}+e_{0}^{\prime}+\frac{H}{2} x_{\phi(1)}
\end{aligned}
$$

For $m=1$, there are $H^{2}$ Golay sequences of length $2^{m}$ over $\mathbb{Z}_{H}$ of this form, and for $m>1$, there are $\frac{H^{m+1} m \text { ! }}{2}$ such sequences.

Paterson, who in [4] generalized the results of Davis and Jedwab ([13]), used an inductive proof for this theorem, which - while less concise than that of Davis and Jedwab - suggests that standard Golay sequences can be seen as arising from an "iterative construction method applied to Golay complementary pairs of length 2 ". The proof of Theorem 3.13 in [10] is, in a way, an extension of this idea, because it uses the three stage process of Fiedler, Jedwab, and Parker discussed above. I won't discuss the details of the proof, but just the general outline.

Stage 1: Clearly, the sequences $\mathbf{a}$ and $\mathbf{b}$ whose algebraic normal forms are $a\left(x_{1}, x_{2}, \ldots, x_{m}\right)$ and $b\left(x_{1}, x_{2}, \ldots, x_{m}\right)$ are those that, for all $\left(x_{1}, x_{2}, \ldots, x_{m}\right) \in \mathbb{Z}_{2}^{m}$, satisfy

$\mathbf{a}\left(x_{m}+2 x_{m-1}+\cdots+2^{m-1} x_{1}\right)$ $=\frac{H}{2} \sum_{k=1}^{m-1} x_{\phi(k)} x_{\phi(k+1)}+\sum_{k=1}^{m} e_{k} x_{\phi(k)}+e_{0}$

$\mathbf{b}\left(x_{m}+2 x_{m-1}+\cdots+2^{m-1} x_{1}\right)$ $=\frac{H}{2} \sum_{k=1}^{m-1} x_{\phi(k)} x_{\phi(k+1)}+\sum_{k=1}^{m} e_{k} x_{\phi(k)}+e_{0}^{\prime}+\frac{H}{2} x_{\phi(1)}$

Using Theorem 2.33, with input pairs $\mathcal{A}_{k}=(0)$ and $\mathcal{B}_{k}=(0)$ for all $k=0,1, \ldots, n$, we get arrays $\mathcal{F}_{n}$ and $\mathcal{G}_{n}$ of size $\mathbf{1}^{(n+1)} \times \mathbf{2}^{(n)}$ over $\mathbb{Z}_{H}$. Remove all dimensions of size 1 . We get $\mathcal{F}^{\prime}=F^{\prime}\left[x_{1}, \cdots, x_{n}\right]$ and $\mathcal{G}^{\prime}=G^{\prime}\left[x_{1}, \cdots, x_{n}\right]$ of size $\mathbf{2}^{(n)}$ over $\mathbb{Z}_{H}$, which, for all $\left(x_{1}, x_{2}, \ldots, x_{n}\right) \in \mathbb{Z}_{2}^{n}$, satisfy

$$
F^{\prime}\left[x_{1}, \cdots, x_{n}\right]=\sum_{k=1}^{n-1} \frac{H}{2} x_{k} x_{k+1}
$$

$$
G^{\prime}\left[x_{1}, \cdots, x_{n}\right]=\sum_{k=1}^{n-1} \frac{H}{2} x_{k} x_{k+1}+\frac{H}{2} x_{1}
$$

So $\mathcal{F}^{\prime}$ and $\mathcal{G}^{\prime}$ form a Golay array pair.

The second step is to use Theorem 2.34, and take affine offsets. Thus, we know that for all $e_{k}, e_{0}^{\prime} \in \mathbb{Z}_{H}$, 


$$
\begin{gathered}
k=0,1, \ldots, n, \\
f^{\prime}\left[x_{1}, \cdots, x_{n}\right]=\sum_{k=1}^{n-1} \frac{H}{2} x_{k} x_{k+1}+\sum_{k=1}^{n} x_{k} i_{k}+e_{0} \\
g^{\prime}\left[x_{1}, \cdots, x_{n}\right]=\sum_{k=1}^{n-1} \frac{H}{2} x_{k} x_{k+1}+\sum_{k=1}^{n} x_{k} i_{k}+e_{0}^{\prime}+\frac{H}{2} x_{1}
\end{gathered}
$$

Then $\left(f^{\prime}\left[x_{1}, \cdots, x_{n}\right]\right)$ and $\left(g^{\prime}\left[x_{1}, \cdots, x_{n}\right]\right)$ form a Golay array pair of size $\mathbf{2}^{(n)}$ over $\mathbb{Z}_{H}$. The third stage requires more results given in [10], and so we won't include all the steps here. It should be fairly clear, however, that these arrays can be mapped to the Golay sequence pairs of Theorem 3.13.

Despite the advantages of the Reed Muller code, this construction does not guarantee a low code rate.

Later on, when we consider complementary sets and multiple L-shift sequences, we will discuss the ways they have been used to further the ideas in this section. For now, however, we will turn to non-Standard Golay sequences.

\subsection{Non-Standard Golay Sequences}

Until Li and Chu's 2005 paper [9], it was believed that standard Golay sequences were the only Golay sequences that existed for lengths $2^{m}$ over $\mathbb{Z}_{2^{k}}$. However, through exhaustive computer searches, Li and Chu found 1024 quaternary Golay sequences of length 16 that could not be produced by Theorem 3.31. This discovery prompted investigation into the structure of this family of Golay sequences (see [6] and [14]), and later to other families of non-standard Golay sequences (see [12]).

Lemma 3.4.1 Let $\mathcal{A}=\left(A\left[i_{1}, \cdots, i_{r}\right]\right)$ be an $n_{1} \times n_{2} \times \cdots \times n_{r}$ array over $\mathbb{Z}_{H}$. Let $\mathcal{A}^{\prime}=\left(A^{\prime}\left[i_{1}, \cdots, i_{r}\right]\right)$ where $A^{\prime}\left[i_{1}, \cdots, i_{r}\right]=A\left[i_{1}, \cdots, i_{r}\right]+c ; c \in \mathbb{Z}_{H}$, and let $\mathcal{A}^{*}=\left(A^{*}\left[i_{1}, \cdots, i_{r}\right]\right)$ be as usual. Then

$\mathcal{C}_{\mathcal{A}}\left(u_{1}, \cdots, u_{r}\right)=\mathcal{C}_{\mathcal{A}^{\prime}}\left(u_{1}, \cdots, u_{r}\right)=\mathcal{C}_{\mathcal{A}^{*}}\left(u_{1}, \cdots, u_{r}\right)$,

$\forall\left(u_{1}, \cdots, u_{r}\right) \neq(0, \cdots, 0)$

Proof: Let $\left(u_{1}, \cdots, u_{r}\right) \neq(0, \cdots, 0)$

$\mathcal{C}_{\mathcal{A}^{\prime}}\left(u_{1}, \cdots, u_{r}\right)$

$=\sum_{i_{1}} \cdots \sum_{i_{r}} \mathcal{E}^{A\left[i_{1}, \cdots, i_{r}\right]+c-A\left[i_{1}+u_{1}, \cdots, i_{r}+u_{r}\right]-c}$

$=\sum_{i_{1}} \cdots \sum_{i_{r}} \mathcal{E}^{A\left[i_{1}, \cdots, i_{r}\right]-A\left[i_{1}+u_{1}, \cdots, i_{r}+u_{r}\right]}$

$=\mathcal{C}_{\mathcal{A}}\left(u_{1}, \cdots, u_{r}\right)$

$\mathcal{C}_{\mathcal{A}^{*}}\left(u_{1}, \cdots, u_{r}\right)$

$\begin{aligned}= & \sum_{i_{1}} \cdots \sum_{i_{r}} \mathcal{E}^{-A\left[n_{1}-1-i_{1}, \cdots, n_{r}-1-i_{r}\right]} \\ & +A\left[n_{1}-1-i_{1}+u_{1}, \cdots, n_{r}-1-i_{r}+u_{r}\right]\end{aligned}$

$=\sum_{i_{1}} \cdots \sum_{i_{r}} \mathcal{E}^{A\left[i_{1}, \cdots, i_{r}\right]-A\left[i_{1}+u_{1}, \cdots, i_{r}+u_{r}\right]}$
$=\mathcal{C}_{\mathcal{A}}\left(u_{1}, \cdots, u_{r}\right)$

Let $\mathcal{A}=\left(A\left[i_{1}, \cdots, i_{r}\right]\right)$ be an $n_{1} \times n_{2} \times \cdots \times n_{r}$ array over $\mathbb{Z}_{H}$.

$E(\mathcal{A})$

$:=\left\{\left(A\left[i_{1}, \cdots, i_{r}\right]+c \mid c \in \mathbb{Z}_{H}\right)\right\} \cup\left\{\left(A^{*}\left[i_{1}, \cdots, i_{r}\right]+c\right) \mid c \in \mathbb{Z}_{H}\right\}$

All arrays in $E(\mathcal{A})$ have identical autocorrelation functions. The number of arrays in $E(\mathcal{A})$ is $H$ or $2 H$, if $A\left[i_{1}, \cdots, i_{r}\right]+c=A^{*}\left[i_{1}, \cdots, i_{r}\right]$ for some $c \in \mathbb{Z}_{H}$ or not, respectively.

This construction allows us to formulate the autocorrelation crossover property, which is how the aforementioned nonstandard quaternary Golay sequences of length 16 are produced.

Suppose $\mathcal{A}, \mathcal{A}^{\prime}, \mathcal{B}$, and $\mathcal{B}^{\prime}$ are arrays and that $\mathcal{C}_{\mathcal{A}}\left(u_{1}, \cdots, u_{r}\right)=\mathcal{C}_{\mathcal{A}^{\prime}}\left(u_{1}, \cdots, u_{r}\right)$ and $\mathcal{C}_{\mathcal{B}}\left(u_{1}, \cdots, u_{r}\right)=$ $\mathcal{C}_{\mathcal{B}^{\prime}}\left(u_{1}, \cdots, u_{r}\right)$ for all $\left(u_{1}, \cdots, u_{r}\right) \neq(0, \cdots, 0)$. Additionally, suppose that $E(\mathcal{A}) \neq E\left(\mathcal{A}^{\prime}\right), E(\mathcal{B}) \neq E\left(\mathcal{B}^{\prime}\right)$, and that $(\mathcal{A}, \mathcal{B})$ and $\left(\mathcal{A}^{\prime}, \mathcal{B}^{\prime}\right)$ are Golay array pairs. Then $\left(\mathcal{A}, \mathcal{A}^{\prime}\right)$ are called a cross over pair. The cross over property then applies - in other words, we know that any $\mathcal{F} \in E(\mathcal{A})$ and $\mathcal{G} \in E\left(\mathcal{B}^{\prime}\right)$ form a Golay pair. Similarly, any $\mathcal{F} \in E\left(\mathcal{A}^{\prime}\right)$ and $\mathcal{G} \in E(\mathcal{B})$ form a Golay pair.

We have seen that standard Golay sequences can be generated using the three stage process with trivial inputs in the first stage. Quaternary nonstandard Golay sequences of length $2^{m}$ can be produced using the three stage process - but their inputs are not trivial, but rather must come from the following cross-over sequences:

Example 3.4.2 (Thm 12) Let $a=$ $\left(\begin{array}{llllllll}0 & 0 & 0 & 2 & 0 & 0 & 2 & 0\end{array}\right)$ and $b=\left(\begin{array}{llllllll}0 & 1 & 1 & 2 & 0 & 3 & 3 & 2\end{array}\right)$. Then each of the pairs in the following set is a cross over pair.

$\left\{(a, b) ;\left(a^{*}, b\right) ;\left(a, b^{*}\right) ;\left(a^{*}, b^{*}\right) ;(b, a) ;\left(b, a^{*}\right) ;\left(b^{*}, a\right) ;\left(b^{*}, a^{*}\right)\right\}$

Additionally, all quaternary Golay cross over pairs of length 8 are formed by affine offsets of these pairs (see [14]).

\section{FURTHER EXTENSIONS OF GOLAY COMPLEMENTARY SEQUENCES}

\subsection{Complementary Sets}

Definition 4.1.1 Let $\mathcal{A}_{1}, \mathcal{A}_{2}, \ldots, \mathcal{A}_{n}$ be $n n_{1} \times n_{2} \times$ $\cdots \times n_{r}$ arrays over $\mathbb{Z}_{H}$. Then they are called a Golay complementary set if

$$
\sum_{i=1}^{n} \mathcal{C}_{\mathcal{A}_{i}}\left(u_{1}, \cdots, u_{r}\right)=0
$$

One of the earlier and more significant papers on Golay complementary sets of sequences is [3]. 
Theorem 4.1.2 [3] A binary complementary set of sequences contains an even number of sequences.

Theorem 4.1.3

Let $\left\{\mathcal{A}_{i}=A_{i}\left[i_{1}, \cdots, i_{r}\right] \mid i=1,2, \ldots, n\right\}$ be a Golay complementary set of $n n_{1} \times n_{2} \times \cdots \times n_{r}$ arrays over $\mathbb{Z}_{H}$. Let $e_{k}, b_{j} \in \mathbb{Z}_{H}, k=0,1, \ldots, r, j=1,2, \ldots n$.

$$
A_{i}^{\prime}\left[i_{1}, \cdots, i_{r}\right]=A_{i}\left[i_{1}, \cdots, i_{r}\right]+\sum_{k=1}^{r} e_{k} i_{k}+b_{i}
$$

Then replacing any $\mathcal{A}_{i}$ with $\mathcal{A}_{i}^{*}=\left(A_{i}{ }^{*}\left[i_{1}, \cdots, i_{r}\right]\right)$ still leaves a complementary set. Additionally, $\left\{\mathcal{A}_{i}^{\prime}=A_{i}^{\prime}\left[i_{1}, \cdots, i_{r}\right] \mid i=1,2, \ldots, n\right\}$ is a Golay complementary set where

$$
A_{i}^{\prime}\left[i_{1}, \cdots, i_{r}\right]=A_{i}\left[i_{1}, \cdots, i_{r}\right]+\sum_{k=1}^{r} e_{k} i_{k}+b_{i}
$$

Proof: We know that

$\mathcal{C}_{\mathcal{A}_{i}}\left(u_{1}, \cdots, u_{r}\right)=\mathcal{C}_{\mathcal{A}_{i}^{*}}\left(u_{1}, \cdots, u_{r}\right)$,

$\forall\left(u_{1}, \cdots, u_{r}\right) \neq(0, \cdots, 0)$

Thus, the first statement in the theorem is trivial. Let $\left(u_{1}, \cdots, u_{r}\right) \neq(0, \cdots, 0)$. Then

$\mathcal{C}_{\mathcal{A}_{i}^{\prime}}\left(u_{1}, \cdots, u_{r}\right)$

$\begin{aligned}= & \sum_{i_{1}} \cdots \sum_{i_{r}} \mathcal{E}^{A_{i}}\left[i_{1}, \cdots, i_{r}\right]+\sum_{k=1}^{r} e_{k} i_{k}+b_{i}-\left(A\left[i_{1}+u_{1}, \cdots, i_{r}+u_{r}\right]\right. \\ & \left.+\sum_{k=1}^{r} e_{k}\left(i_{k}+u_{k}\right)+b_{i}\right)\end{aligned}$

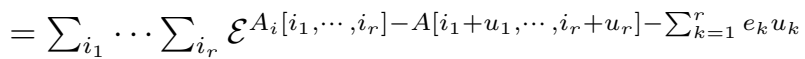

$=\mathcal{E}^{-\sum_{k=1}^{r} e_{k} u_{k}} \mathcal{C}_{\mathcal{A}_{i}}\left(u_{1}, \cdots, u_{r}\right)$

Thus,

$\sum_{i=1}^{n} \mathcal{C}_{\mathcal{A}_{i}^{\prime}}\left(u_{1}, \cdots, u_{r}\right)$

$=\sum_{i=1}^{n} \mathcal{E}^{-\sum_{k=1}^{r} e_{k} u_{k}} \mathcal{C}_{\mathcal{A}_{i}}\left(u_{1}, \cdots, u_{r}\right)$

$=\mathcal{E}^{-\sum_{k=1}^{r} e_{k} u_{k}} \sum_{i=1}^{n} \mathcal{C}_{\mathcal{A}_{i}}\left(u_{1}, \cdots, u_{r}\right)$

$=0$

This proves the second part of the statement.

Claim 4.1.4 A sequence which is a member of Golay complementary set of size $k$ where each sequence is of length $n$ has PMEPR less than $k$.

Proof: Let $\left\{a_{i} \mid i=1,2, \ldots k\right\}$ and be a Golay complementary set. So we have

$$
\sum_{i=1}^{k} \mathcal{C}_{a_{i}}(u)=0
$$

Thus,

$$
\sum_{i=1}^{k} P_{a_{i}}(t)=k n+\sum_{u \neq 0}\left[\mathcal{E}^{-H u \triangle f t} \sum_{i=1}^{k} \mathcal{C}_{a_{i}}(u)\right]=k n
$$

As $P_{a_{i}}(t)=\left|s_{a_{i}}(t)\right|^{2} \geq 0, P_{a_{i}}(t) \leq k n$. So the PMEPR of $a_{i}$ is less than $k$.

Because the code rate of standard Golay sequences rapidly approaches 0 as the length of the sequences increases, it can be advantageous to use Golay complementary sets that are related to Reed-Muller Codes in a similar way. See Theorem 1 in [18] for a family of Golay complementary sets. For more information, see [4], [15], and [18].

\subsection{Multiple L-Shift Complementary Sequences}

Multiple L-shift complementary sequences were introduced as an alternative to Golay complementary sets as a way of increasing the code rate of standard sequences.

Definition 4.2.1 Let $a$ and $b$ be $H$-phase sequences of length $n$, and $L \in \mathbb{Z}$. If

$$
\mathcal{C}_{a}(u)+\mathcal{C}_{b}(u)=0, \text { for } u=0 \bmod L, u \neq 0
$$

Then $a$ and $b$ are said to be a multiple L-shift complementary sequence pair.

I have extended this definition to arrays.

Definition 4.2.2 Let $\mathcal{A}$ and $\mathcal{B}$ be $n_{1} \times n_{2} \times \cdots \times n_{r}$ $H$-phase arrays and $L \in \mathbb{Z}$. If

$\mathcal{C}_{\mathcal{A}}\left(u_{1}, \cdots, u_{r}\right)+\mathcal{C}_{\mathcal{B}}\left(u_{1}, \cdots, u_{r}\right)=0$

for $\left(u_{1}, \cdots, u_{r}\right)=(0, \ldots, 0) \bmod L,\left(u_{1}, \cdots, u_{r}\right) \neq$ $(0, \ldots, 0)$

Then $\mathcal{A}$ and $\mathcal{B}$ are multiple L-shift complementary array pair.

This is a broader definition than that for Golay array pairs. As for Golay array pairs, replacing a multiple Lshift complementary array pair $\mathcal{A}$ and $\mathcal{B}$ with $\mathcal{A}^{*}$ and $\mathcal{B}^{*}$ or with affine offsets of $\mathcal{A}$ and $\mathcal{B}$ does not change the complementary property.

Claim 4.2.4 [Theorem 1, 7] A sequence which is a member of multiple L-shift complementary pair has PMEPR less than $2 L$.

Theorem 4.2.5 [Theorem 2, 7] Let $L=2^{d}$, $\phi$ a permutation of $\{1,2, \ldots, m-d\}$, and $e_{k, l}, e_{l}^{\prime} \in \mathbb{Z}_{H}$. Let

$f\left(x_{1}, x_{2}, \ldots, x_{m}\right)$

$=\frac{H}{2} \sum_{k=1}^{m-d} x_{\phi(k)} x_{\phi(k+1)}$

$+\sum_{k=1, k \neq l}^{m} \sum_{l=m-d+1}^{m} e_{k, l} x_{k} x_{l}+\sum_{l=1}^{m} e_{l} x_{l}$

Let $e, e^{\prime} \in \mathbb{Z}_{H}$ Then the sequences $\mathbf{a}$ and $\mathbf{b}$ whose algebraic normal forms are $a\left(x_{1}, x_{2}, \ldots, x_{m}\right)$ and $b\left(x_{1}, x_{2}, \ldots, x_{m}\right)$ respectively form a multiple L-shift complementary sequence pair, where

$$
\begin{gathered}
a\left(x_{1}, x_{2}, \ldots, x_{m}\right)=f\left(x_{1}, x_{2}, \ldots, x_{m}\right)+e \\
b\left(x_{1}, x_{2}, \ldots, x_{m}\right)=f\left(x_{1}, x_{2}, \ldots, x_{m}\right)+e^{\prime}+2^{h-1} x_{\phi(1)}
\end{gathered}
$$

Clearly, these are related to standard Golay sequences.

There are a number of known results on the possible lengths of multiple L-shift complementary sequence pairs. For example, they exist for length $n$ where $L \leq$ $n \leq 2 L$, and the length must be the sum of at most $2 \mathrm{~L}$ squares. For the proofs of these and other results, see [11]. 


\section{CONCLUSION}

Golay complementary sequences, from their beginning in Golay's paper, have been developed and generalized considerably. It is interesting to see the developments that have occurred over the years. Golay's ideas have been generalized from pairs of binary sequences to sets of arrays with entries over $\mathbb{Z}_{H}$. Questions in the area focus on possible lengths of Golay sequences, their enumeration, and methods of construction, particularly within the newer the constructions of Golay arrays and Golay sets. In this paper, we have focused on the applications within coding theory of Golay complementary sequences, because much of the development of the theory of Golay sequences has been within this context. However, Golay complementary sequences have been used in many fields, including in Ising spin systems (physics), infrared spectrometry, Hadamard matrices, and various communications systems [19]. Thus, they have both important applications, as well as involving interesting mathematics.

\section{REFERENCES}

1. W. H. Holzmann, H. Kharagani, "A Computer Search for Complex Golay Sequences", Australasian Journal of Combinatorics, pp.251-258, 10 (1994) 4,10

2. M. J. E. Golay, "Complementary Series," IRE Trans. Inform. Theory, IT-7, pp. 82-87, Apr. 1961.

3. C. C. Tseng, C. L. Liu, "Complementary sets of sequences," IEEE Trans. Inform. Theory, IT-18, no. 5, pp. 644-651, Sept. 1972

4. K. G. Paterson, "Generalized Reed-Muller codes and power control in OFDM modulation", IEEE Trans. Inform. Theory, IT-46, no. 1, 104-120, Jan. 2000

5. J. Jedwab, M. Parker, "Golay complementary array pairs", Designs, Codes, and Cryptography, Vol 44, Sept. 2007

6. F. Fiedler, J. Jedwab, "How Do More Golay Sequences Arise", IEEE Trans. Inform. Theory, Vol. 52, 42614266, May 2005

7. W. Chen, C. Tellambura, "Identifying a Class of Mul- tiple Shift Complementary Sequences in the Second Order Cosets of the First Order Reed-Muller Codes", Communications, Vol. 1, 618-721, 2005

8. D. Matiae, "OFDM as a possible modulation technique for multimedia applications in the range of $\mathrm{mm}$ waves", Introduction to OFDM, II Edition, October 1998

9. Y. Li and W. B. Chu, "More Golay sequences", IEEE Transactions on Information Theory, vol. 51, no. 3, pp. 1141-1145, 2005.

10.F. Fiedler, J. Jedwab and M.G. Parker, A multidimensional approach to the construction and enumeration of Golay complementary sequences, J. Combinatorial Theory (Series A), 2007.

11. Y. Xin and I. Fair, Multiple L-shift Complementary Sequences, IEICE Trans. Fundamentals, Vol.E89-A, No.10 October 2006

12. F. Fiedler, J. Jedwab, and A. Wiebe, A new source of seed pairs for Golay sequences of length $2 \mathrm{~m}$, J. Combinatorial Theory (Series A), 117 (2010), 589-597

13. J . A. Davis and J. Jedwab, Peak-to-mean power control in OFDM, Golay complementary sequences, and Reed-Muller codes, IEEE Trans .Inform. Theory, vol. 45. pp. 2397-2417. Nov. 1999.

14.Y. Li and Y. C. Kao, "Structures of Non-GDJ Golay Sequences," Proceedings of the 2005 International Symposium on Information Theory (ISIT 2005), pp. 378381, Adelaide, Australia, Sept. 2005.

15. Schmidt, K.-U.; , "Complementary Sets, Generalized Reed-Muller Codes, and Power Control for OFDM," Information Theory, IEEE Transactions on , vol.53, no.2, pp.808-814, Feb. 2007

16. R. Turyn and J. Storer, "On Binary Sequences", Proceedings of the American Mathematical Society , Vol. 12, No. 3 (Jun., 1961), pp. 394-399

17. Eliahou S., Kervaire M., Saffari B., On Golay polynomial pairs. Adv. Appl. Math. 12, 235-292 (1991).

18. Schmidt, K.-U., "Complementary Sets, Generalized Reed-Muller Codes, and Power Control for OFDM," Information Theory, IEEE Transactions on , vol.53, no.2, pp.808-814, Feb. 2007

19. M. Parker, K. Paterson, C. Tellambura, "Golay Complementary Sequences", Jan. 2004. http://www.isg.rhul.ac.uk/ ${ }^{\wedge} \mathrm{kp} /$ golaysurvey.pdf 